\title{
Improving CAD/CAM Process Chains in Forging Industries in the Era of Digitalization Based on a Case Study
}

\author{
Markus Brillinger ${ }^{1}$, Juan Calejero Martinez ${ }^{2}$, Johannes Schmid ${ }^{3}$ \\ ${ }^{1}$ Pro2Future \\ Inffeldgasse 25F, $8010 \mathrm{Graz}$, Austria \\ markus.brillinger@pro2future.at \\ ${ }^{2}$ Escuela de Ingeniería y Arquitectura University of Zaragoza \\ Calle María de Luna, 3, Zaragoza, Spain \\ 620474@unizar.es \\ ${ }^{3}$ Institute of Production Engineering, Graz University of Technology \\ Inffeldgasse 25F, $8010 \mathrm{Graz}$, Austria \\ johannes.schmid@tugraz.at
}

\begin{abstract}
Currently, in today's market, there is a wide spectrum of CAD/CAM software systems. Choosing the right software system out of that huge variety could be a challenging task to perform. The ideal decision-making procedure should not only take into intern functionality aspects into consideration, but also the potential of the chosen software system in activities related to digitalization like Digital Twins or Tool Management Libraries. In this paper a decision-making procedure is proposed and applied in a forging company for choosing a CAD/CAM software system that could enhance the process chain in the era of digitalization.
\end{abstract}

Keywords: Computer Aided Design (CAD), Computer Aided Manufacturing (CAM), Analytical Hierarchy Process (AHP) method, Decision-making, Improving CAD/CAM process chain, Forging industry, Digitalization.

\section{Introduction}

While increasing computer capacity, the industrial product design process is changing continuously: powerful Computer-Aided Design systems (CAD) enable simulation-based design even in early stages of product design and machineadapted Computer-Aided Manufacturing systems (CAM) provide off-line programming of the manufacturing procedure.

Since digitalization progresses, process chains (from CAD to machine) are becoming virtualized the question of how far the process chains are following the trend towards digitalization may determine the competitiveness and thus the survival of a company in the future.

Based on a case study in the forging die making department of a Styrian medium-sized forging company, it will be examined how the current CAD/CAM infrastructure can be optimized with respect to the trend towards digitalization. In the chapter State of the Art it is shown, which methods already exist for the selection of suitable CAD/CAM systems, which requirements are placed on such systems in forging die making of a forging operation and the current CAD/CAM situations in different forging companies will also be discussed. The chapter Research gap elaborates on the questions this paper is dealing with. The chapter Methodology describes the current situation in the forging die making department in the investigated Styrian forging company, presents future scenarios with respect to digitalization and discusses validation criteria for the scenarios. The chapter Results deals with the findings and in the chapter Conclusion/Future work future steps in the Styrian forging company are recommended.

\section{State of the Art}

CAD and CAM software systems constitute a basic technology in today's industry. Nowadays, in current market, there are many different CAD and CAM software systems. As a result, finding a software system that suits the requirements among this wide variety can be a difficult question to answer. In order to solve this problem, there are some methodologies that could facilitate the decision-making process.

After an intensive literature research, the most common procedure for CAD/CAM software system decision-making was found to be the Analytical Hierarchy Process (AHP) method [1]. In the research done by [2], the AHP method was used 
as a decision-making technique for identifying and prioritizing important factors for CAD/CAM software system selection. In another research [3], authors point out that the application of AHP method, in CAD/CAM decision-making, improves the team decision-making process and reduces the amount of time required. Nevertheless, this method includes some disadvantages like the uncertainty on evaluation, which makes the AHP method imprecise to capture the right judgment, also it deals with an unbalanced scale of judgment and the subjective opinion has a great influence on the results too [4].

The CAD/CAM decision-making process from the case study was made for a forging company. As a first approach, the CAD/CAM situation of the direct competitors was checked. Nine companies were identified as direct competitors, some were located in Belgium, Slovenia and Germany but, most of them were on Austrian territory. These nine competitors' situation is presented in Figure 1. In this bubble diagram it is possible to deduct the size of the company from the radius of the circle; the bigger the radius, the more employees there are in the company.

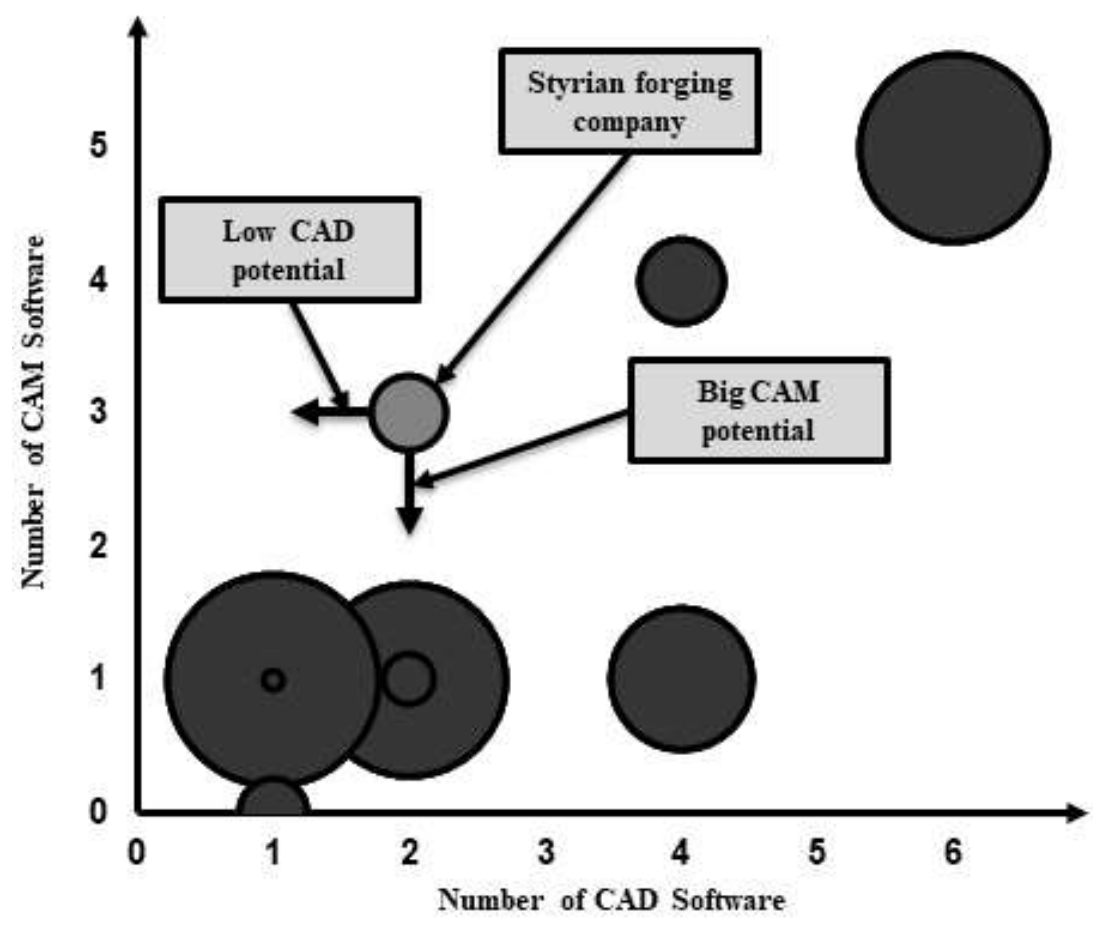

Fig. 1: Competitors analysis.

Based on the competitors' software infrastructure, it was seen that the room for improvement relied on reducing the amount of CAx systems to a minimum. The ideal solution would be one system for each purpose, one for CAD and one for CAM. Due to the prevalent lack of suitable full data exchange mechanisms at the interface between CAD and CAM, this solution would avoid the information loss produced for using native data formats like .STEP and .IGES [5]. Moreover, this solution would adapt the scheme of the Styrian forging company towards the implementation of future digitalization processes like Digital Twin simulation, Tool Management Libraries, Machine Code Based Simulation, CAD and CAM templates, Feature-Macro Mapping, Application Programming Interfaces (API) and virtual CNC control.

Currently, the digitalization trend, regarding processes associated with CAD and CAM, focuses on Digital Twins. These Digital Twins enhance many different areas in a technological environment like production planning, predictive maintenance and quality assurance [6,7]. More specifically, Digital Twins are a suitable solution for aiding machining equipment like mills and lathes [8].

Regarding the forging die making process, the process is based on the inverse design procedure. Starting from the should-geometry of the forged part, development work proceeds to determine the geometry of the different forming and pre-forming dies. This procedure of design is highly related to the use of computer aided technologies (CAx). These 
tools made possible to push out the trial and error procedure, used in conventional die forging, and led to a significant reduction of time, energy and material waste on design and manufacturing stages of forging dies [9].

The forging die making process at the Styrian forging company could be briefed in Figure 2.

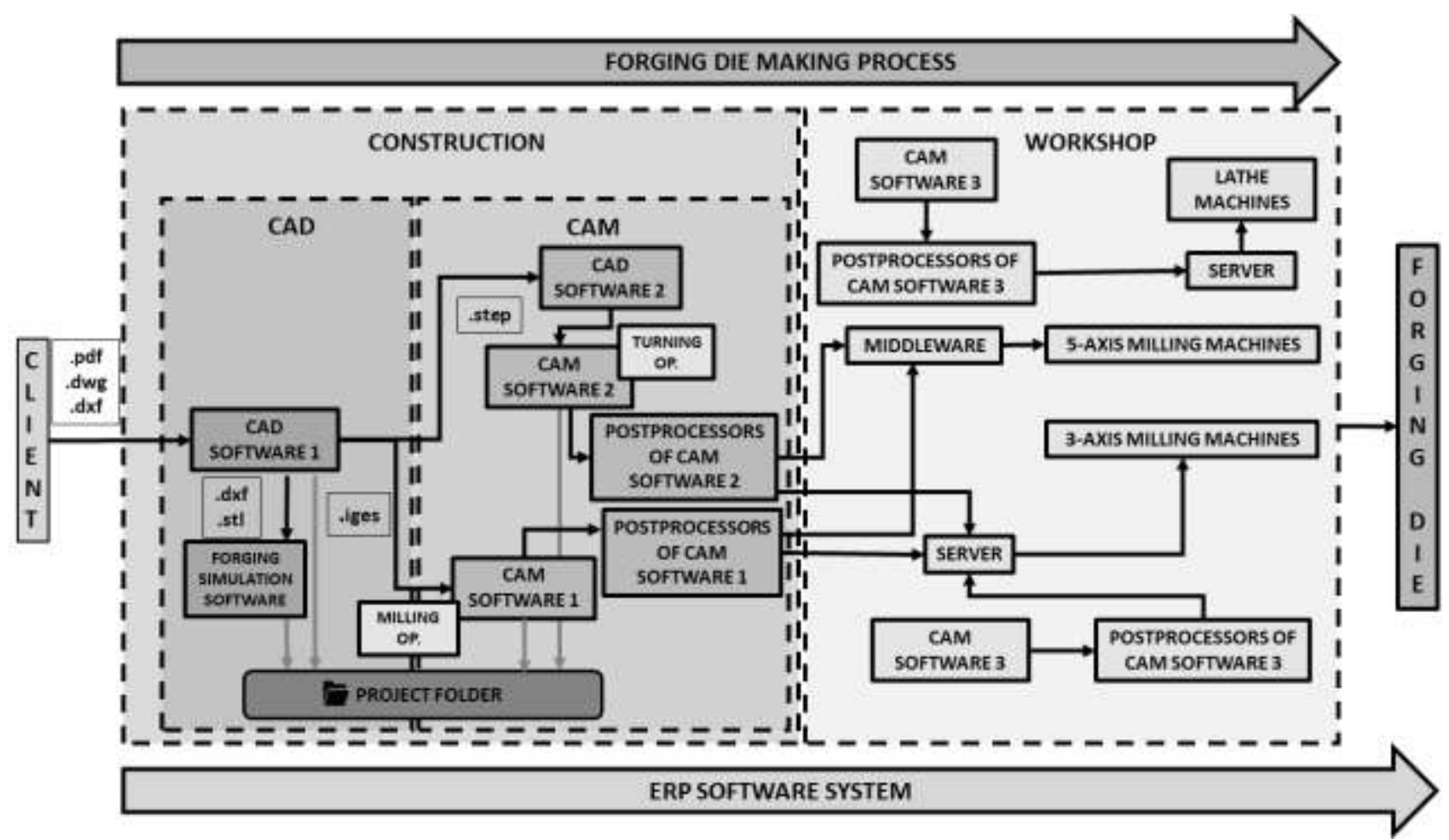

Fig. 2: Current forging die making process from case study.

The information of the forging product is provided by the client to the forging die making department as .PDF, .DWG or .DXF. At the CAD department, the designers create the forging die out of the forging product with CAD Software 1. After that, the design is simulated in the Forging Simulation Software.

When the design is completed and the forging simulation process seems to be feasible, the data from the CAD department are sent to the CAM department. There, CAM programmers use CAM Software 1 and CAM Software 2 to generate the NC Code for the machines.

When starting with CAM Software 2, data are imported as .STEP format, but as there is no direct link between CAD Software 1 and CAM Software 2, the CAD files need to be imported through CAD Software 2 where some design modifications are made. Then, CAM Software 2 is opened directly through CAD Software 2 interface.

In the other way, when starting with CAM Software 1, data are imported as .IGES format. Generally, CAM Software 1 is rather intended for milling operations and complex operations. CAM Software 2 is rather intended for easy tasks, such as feature recognition functionalities and some turning operations.

Once the NC-Codes are generated, the data are sent to the shopfloor. Depending on which machine is required, the importing process to the machine is done via Middleware or via Server. Some pieces might require an additional task, which is programmed at the shopfloor using CAM Software 3. When forging dies are ready, they are sent to the production line where they are used to shape final forged products.

Summing up, the current forging die making process requires two CAD software systems and three CAM software systems.

\section{Research gap}

As already mentioned before in the chapter State of the Art, the AHP method includes a high level of subjectivity inside its procedure therefore results could lack of an objective point of view. Additionally, due to the singularity of the 
requirements from $\mathrm{CAD} / \mathrm{CAM}$ process chains in forging industry, there are no publications available dealing with this specific topic in relation to digitalization.

Summing up, the following gaps emerged:

- Current AHP method, used in many CAD/CAM decision-making procedures, has drawbacks towards making the right decision.

- There are no publications available regarding the improvement of CAD/CAM process chains particularly in forging industry in relation to digitalization.

\section{Methodology}

This chapter is divided in two separate parts. The first part presents the future possible scenarios, considering each combination between the current software systems and a certain amount of software system alternatives. The second part, deals with the criteria and decision-making to evaluate those previously mentioned scenarios with respect to digitalization.

\subsection{Scenario definition}

The CAD/CAM software system selection method used in the presented case study, evaluates whether the current software scenario could be improved in CAD and/or in CAM with some software system alternatives. These alternatives are CAD Alternative, CAM Alternative 1 and CAM Alternative 2. CAD Alternative and CAM Alternative 2 are provided by the same software supplier. Additionally, this supplier offers a middleware solution and an ERP software system which is commonly widespread. Therefore, considering the combination of current software systems and alternative software systems, there would be a total of five different future scenarios to evaluate, as presented in Table 1.

Table 1: Possible future scenarios.

\begin{tabular}{|l|l|}
\hline \multicolumn{2}{|c|}{ CAD/CAM combination } \\
\hline Scenario 1 & CAD Software 1 $\rightarrow$ CAM Alternative 1 \\
\hline Scenario 2 & CAD Software 1 $\rightarrow$ CAM Alternative 2 \\
\hline Scenario 3 & CAD Alternative $\rightarrow$ CAM Alternative 1 \\
\hline Scenario 4 & CAD Alternative $\rightarrow$ CAM Alternative 2 \\
\hline Scenario 5 & CAD Alternative - \\
\hline & \\
\hline CAM Software 1 \\
\hline
\end{tabular}

\subsection{Scenario evaluation}

The potential for improvement, as previously mentioned, relies on reducing the number of CAx systems in the current scenario, especially in CAM, in order to decrease the expenditure of file transfer and adapting the software infrastr ucture for future digitalization technologies like simulation of Digital Twin, robots, laser welding and additive manufacturing. 
Regarding the criteria to evaluate the five scenarios, they are divided in two different categories: black box criteria and white box criteria.

In relation to the black box criteria, each scenario is evaluated objectively by its external background, and not by its own functionalities. These criteria evaluate the amount of software systems, resellers, interfaces and postprocessors needed in the scenario.

In relation to the white box criteria, each future scenario is evaluated objectively in terms of the functionalities from each software system that composes it. These criteria comprise: a function-based comparison to check if the scenario covers all the functionalities presented in the current software scenario, Machine Code Based Simulation criterion, Simulation of Digital Twin criterion, Tool Management Library criterion, CAM Templates criterion, Connectivity with ERP Software System criterion, Feature-Macro Mapping criterion, Application Programming Interface criterion. Moreover, it includes a subjective side, the opinion-based comparison. For this criterion a simplified version of the AHP method is implemented. This simplified version of the AHP method analyses the opinion of the Styrian forging company's experts regarding the software tools included in CAD Alternative, CAM Alternative 1 and CAM Alternative 2.

These criteria are summed up in Table 2.

Table 2: Black box and white box criteria.

\begin{tabular}{|c|c|}
\hline Black box criteria & White box criteria \\
\hline Number of software systems & Function-based comparison \\
Number of software resellers & Machine Code Based Simulation \\
Number of interfaces & Simulation of Digital Twin \\
Number of postprocessors & Tool Management Library \\
& CAM Templates \\
& Connectivity with ERP Software System \\
& Feature-Macro Mapping \\
& Application Programming Interface \\
& Opinion-based comparison \\
\hline
\end{tabular}

\section{Results}

Regarding the black box criteria, research showed that Scenario 1, Scenario 2, Scenario 3 and Scenario 4 were at the same level of preference whereas Scenario 5 required the highest amount of software systems, software resellers, software interfaces and postprocessors. Therefore, Scenario 5 seems to be the least efficient one basing on its external background.

Regarding the white box criteria, according to the ongoing digitalization influence, there are a lot of challenges that a CAM software system has to deal with.

To enable a correct simulation, a digital copy of the machine, tools, work piece, raw material and clamping elements is required. In order to guarantee this in a multi-machine setting in which many people work, a Tool Management Library is necessary, to ensure that all digital tools are up to date.

Another important point regarding the possibility of a ghost shift is Machine Code Based Simulation. Based on this simulation, the NC code, which is generated due to the postprocessing of the CAM operations, is simulated for any collisions, which is shown in the CAM department of Figure 3.

Some research has shown that current CAM systems offer different approaches to automation mechanisms, to assist in the creation of CAM operations. In general, the CAM automation mechanisms are CAM Templates, Feature-Macro Mapping and Application Programming Interfaces.

The first option is using CAM Templates. These templates include the application of pre-fabricated CAM operations, which were previously created and tested for similar components to new geometries.

The second option is the Feature-Macro Mapping. In this process, machining features with parameters such as hole diameter, pocket depth and groove-width are assigned to a specific machining sequence via macros. Every feature needs to be defined and compared with a suitable tool, which needs to be used, in order to manufacture it. A challenge in this context is the clear definition of the editing features. 
The third option is the Application Programming Interface (API). API extensions usually have access to the CAD model. They can do any analysis and can perform on the geometry of the CAD file. API extensions can also customize the user interface of the CAM system and integrate with them. In addition, they are able to independently create and parameterize machining operations. However, the extensive possibilities also entail a very high level of care and, in the case of API, high licensing costs.

To remain competitive in the market, these functionalities, Feature-Macro Mapping and Application Programming Interface (API) can be key features.

There are some aspects to be highlighted regarding Scenario 1, 2, 3 and 4.

In relation to the function-based comparison criterion, the most preferred scenarios were those containing CAM Alternative 2, like Scenario 2 and Scenario 4.

The Machine Code Based Simulation, the simulation after the postprocessing works, for the scenarios 1 to 4, as they include CAM Alternative 1 and CAM Alternative 2.

When it comes to the simulation of Digital Twin, Tool Management Library, CAM Templates, the scenarios 1 to 4 provide these functionalities. However, the performance of Scenario 4, according to these functionalities, is remarkable.

Regarding the connectivity with ERP Software System, the scenarios 1 to 4 provide these functionalities.

In relation to Feature-Macro Mapping, this functionality works properly for Scenario 4 but definitely not for Scenario 1, Scenario 2 and Scenario 3, because of the data loss according to the native data transfer.

Application Programming Interface, this option is the most complex option, of automation mechanisms. It works properly on Scenario 4 and with restrictions on Scenario 1, Scenario 2 and Scenario 3.

Concerning the opinion-based comparison criterion (AHP method), the most preferred scenario was Scenario 2 closely followed by Scenario 4, third place was given to Scenario 1 and Scenario 3 at last place.

Summing up, the most preferred scenario would be Scenario 4, which is presented in Figure 3. The implementation of this scenario is discussed in the chapter Conclusion/Future work.

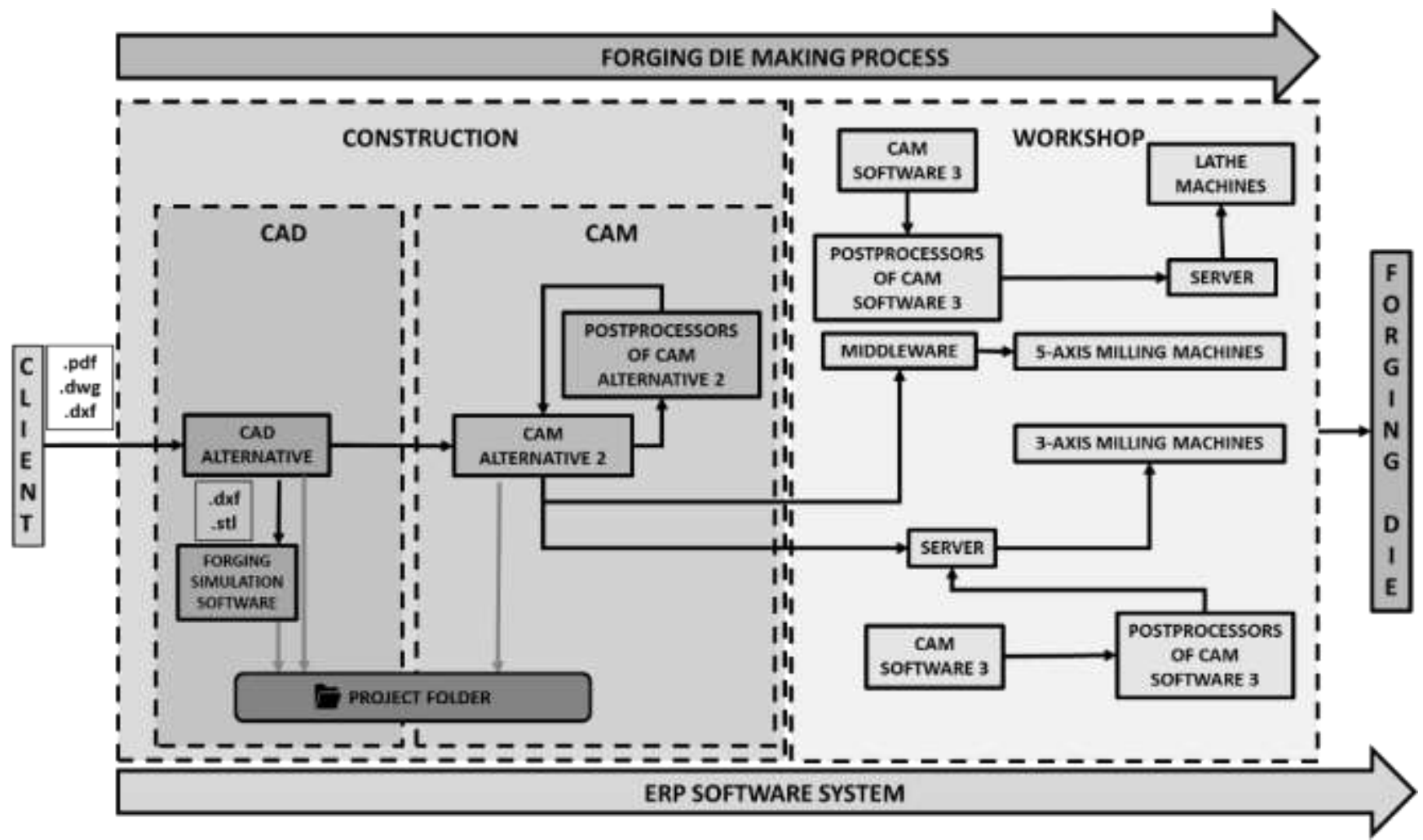

Fig. 3: Forging die making process in Scenario 4. 


\section{Conclusion/Future work}

For an efficient design of the CAD/CAM process chain, it is necessary to identify the steps first, which cause the biggest efforts. It can be seen that the CAM programming, in particular, uses a lot of resources.

As mentioned above, the pressure to perform is enormous today. In order to stay competitive in the market, every possible advantage must be taken. In the chapter Results, the advantages result from Scenario 4 because of some perfectly suitable CAM automation mechanisms and some outstanding functionalities according to the implementation of simulation of Digital Twin, Tool Management Library.

Implementing a system like Scenario 4 is very complex, time consuming and expensive. It is not recommended to realize this scenario in one iteration step, because the daily business would suffer significantly. To avoid this problem, the first step in the implementation of Scenario 4 is the implementation of Scenario 2. As mentioned in chapter Results, Scenario 2 would be the most preferred scenario, if just the aspect of the opinion-based comparison (AHP method) was considered. If Scenario 2 has been realized, the implementation of CAD Alternative can be carried out and so on Scenario 4 can be reached.

The next step after implementing Scenario 4, is the installation of a Tool Management Library. It is important that the realization of this library follows the implementation of CAD Alternative, otherwise changes to the digital tools would not be possible.

To enable further improvements of the NC-Code before testing on the machine, CAM simulations can be extended with a virtual CNC control. The benefit of this possibility is the perfect simulation environment and in the wider sense, the increase in efficiency in the production process.

\section{Acknowledgements}

Pro $^{2}$ Future is funded as part of the Austrian COMET Program-Competence Centers for Excellent Technologies under the auspices of the Austrian Federal Ministry of Transport, Innovation and Technology, the Austrian Federal Ministry for Digital and Economic Affairs, and the Provinces of Upper Austria and Styria. COMET is managed by the Austrian Research Promotion Agency FFG.

\section{References}

[1] T. L. Saaty, "The analytic hierarchy process what it is and how it is used," 4922 Ellsworth Avenue, Pittsburgh, PA 15213, U.S.A: Pergamon Journals Ltd, 1987

[2] G. Zakria, Z. Guan, Y. Riaz, M. Jahanzaib, A. Khan, "Selecting and prioritizing key factors for CAD/CAM software in small and medium-sized enterprises using AHP," Berlin Heidelberg: Higher Education Press and Springer-Verlag, 2010 .

[3] V. P. Pinay, G. Kannan, "Multi-criteria decision-making for the selection of CAD/CAM system," France: SpringerVerlag, Int J Interact Des Manuf, vol. 2, pp. 151-159, 2008.

[4] Z. Ayağ, "CAD software evaluation for product design to exchange data in a supply chain network," Industrial Engineering Department, Kadir Has University Kadir Has Campus, Cibali 34083, Fatih, Istanbul, Turkey: Int. J Sup. Chain. Mgt, 2015.

[5] G. Reinhart, "Handbuch Industrie 4.0 - Geschäftsmodelle, Prozesse, Technik," Carl Hanser Verlag, 2017.

[6] SL Controls. [Online]. Available: https://slcontrols.com/what-is-digital-twin-technology-and-how-can-it-benefitmanufacturing/

[7] Net Objex. [Online]. Available: https://www.netobjex.com/how-can-digital-twin-technology-benefit-yourorganization/

[8] Digitalist. [Online]. Available: https://www.digitalistmag.com/iot/2018/04/23/digital-twin-technology-transformingmill-industry-06090500

[9] M. Milutinović, D. Vilotić, D. Movrin, "Precision forging - tool concepts and process design," Faculty of Technical Sciences, Novi Sad, Serbia. Journal for Technology of Plasticity, vol. 33, no. 1-2, 2008. 